\title{
A relação entre o uso de hipnóticos e sedativos e o desenvolvimento de alterações do humor em estudantes de medicina de uma universidade particular de Sergipe
}

\author{
The relationship between the use of hypnotics and sedatives and the development of \\ mood changes in medical students at a private university in Sergipe
}

La relación entre el uso de hipnóticos y sedantes y el desarrollo de cambios del humor en estudiantes de medicina en una universidad privada en Sergipe

Rafael Ribeiro Almico Fraga1*, Ana Celia Goes Melo Soares ${ }^{1}$, Nathalia Nascimento Santana ${ }^{1}$, Josilda Ferreira Cruz ${ }^{1}$, Mayana Lula Andrade ${ }^{1}$, Rebeca dos Santos Sirqueira ${ }^{1}$, Antônio Souza Lima Júnior ${ }^{1}$.

\section{RESUMO}

Objetivo: Avaliar a relação entre o uso de hipnóticos e sedativos e o desenvolvimento de queixas associadas a alterações do humor em estudantes de medicina de uma universidade particular de Sergipe. Métodos: Trata-se de recorte de estudo transversal, de abordagem analítica qualiquantitativa, conduzido com 281 estudantes de medicina de uma universidade em Sergipe. O uso dessas substâncias foi avaliado por aplicação de um questionário próprio criado para este fim. Os dados foram analisados por estatística descritiva com auxílio do software R Core Team 2020 e o nível de significância adotado foi de $5 \%$. O estudo teve aprovação do Comitê de Ética em Pesquisa. Resultados: Do total de 281 estudantes, 38,1\% já haviam consumido hipnóticos e sedativos, sendo a maioria do sexo feminino, e o principal fator motivador para o uso o estresse. $56,1 \%$ afirmaram diagnóstico prévio de ansiedade e $27,1 \%$, de depressão. Em relação ao desenvolvimento de queixas associadas a alterações do humor após o uso, 38,3\% relataram apresentar-se deprimidos ou desinteressados nas atividades diárias e 50,5\% relataram medo, ansiedade e preocupação excessivos. Conclusão: Evidenciou-se relação entre o consumo de hipnóticos-sedativos e o surgimento de alterações do humor associadas a sintomas depressivos e de ansiedade.

Palavras-chave: Hipnóticos e sedativos, Humor, Depressão, Ansiedade, Estudantes de medicina.

\begin{abstract}
Objective: Establish the relationship between the use of hypnotics and sedatives and the development of complaints associated with mood changes in medical students at a private university in Sergipe. Methods: This is a cross-sectional study with a quali-quantitative analytical approach, conducted with 281 medical students from a university in Sergipe. The use of these substances was assessed by applying a questionnaire created for this purpose. The data were analyzed using descriptive statistics with the aid of the R Core Team 2020 software and the level of significance adopted was 5\%. This study was approved by the Research Ethics Committee. Results: In a total of 281 students, $38.1 \%$ have already used hypnotics and sedatives, the majority being female, and the main motivating factor for use was stress. $56.1 \%$ reported a previous diagnosis of anxiety and $27.1 \%$ reported previous depression. Regarding the development of mood complaints after using these drugs, $38.3 \%$ reported being depressed or uninterested in daily activities and $50.5 \%$ reported excessive fear, anxiety and worry. Conclusion: The relationship between the consumption of hypnotics-sedatives and the emergence of mood changes associated with depressive and anxiety symptoms became evident.
\end{abstract}

Keywords: Hypnotics and sedatives, Mood, Depression, Anxiety, Medical students.

1 Universidade Tiradentes (UNIT), Aracaju-SE, *E-mail: almicorafael@gmail.com SUBMETIDO EM: 5/2020 ACEITO EM: 6/2020

PUBLICADO EM: 8/2020 


\section{RESUMEN}

Objetivo: Establecer la relación entre el uso de hipnóticos y sedantes y el desarrollo de quejas asociadas con cambios del humor en estudiantes de medicina de una universidad privada en Sergipe. Métodos: Es un estudio transversal, analítico, cualitativo cuantitativo, realizado con 281 estudiantes de medicina en una universidad en Sergipe. El uso de estas sustancias se evaluó mediante un cuestionario creado para este propósito. Los datos se analizaron utilizando estadísticas descriptivas con la ayuda del software $\mathrm{R}$ Core Team 2020 y el nivel de significación adoptado fue del $5 \%$. Este estudio fue aprobado por su Comité de Ética en Investigación. Resultados: De 281 estudiantes, el 38.1\% ya ha usado hipnóticos-sedantes, la mayoría son mujeres, y el principal factor de motivación para su uso fue el estrés. El 56.1\% informó un diagnóstico previo de ansiedad y el $27.1 \%$ informó depresión previa. Sobre el desarrollo de quejas del humor después de usar estos medicamentos, el $38.3 \%$ informó estar deprimido o desinteresado en las actividades diarias y el $50.5 \%$ informó temor, ansiedad y preocupación excesivos. Conclusión: La relación entre el consumo de hipnóticossedantes y la aparición de cambios del humor asociadas con síntomas depresivos y de ansiedad se hizo evidente.

Palabras clave: Hipnóticos y sedantes, Humor, Depresión, Ansiedad, Estudiantes de medicina.

\section{INTRODUÇÃO}

A entrada no ensino superior é um marco na vida de um estudante que implica grandes modificações e alta carga estressora. Diversas são as fontes de estresse com que os estudantes se deparam no ambiente universitário. O alto nível de cobrança e a grande carga de estresse a que são submetidos os graduandos em medicinas são justificados pela elevada carga acadêmica, por longas horas de estudo, ambiente muitas vezes adverso, sono insatisfatório, alto índice de competitividade, além de fatores relacionados a vida pessoal (QUEK TT, et al., 2019).

No curso de medicina, os graduandos são submetidos a vários outros momentos estressantes durante sua vida acadêmica, as situações em que o aluno reside sozinho e distante de casa, o período longo e em tempo integral dos cursos, a grande quantidade de informações que precisam adquirir e a influência da atividade acadêmica sobre suas atividades de lazer e relacionamentos sociais. Além disso, o próprio contato dos estudantes com pacientes com doenças graves, sofrimento e morte são importantes fontes de estresse (CANDIDO FJ, et al., 2018; VOLPE U, et al., 2019). Toda essa situação a qual os estudantes são submetidos tem o potencial de produzir efeitos negativos à saúde mental e emocional dos aprendizes, além de interferir na capacidade de aprendizado e qualidade de vida (RAFIQUE N, et al., 2019).

Os estudantes de medicina, submetidos às altas demandas acadêmicas, muitas vezes apresentam ansiedade, depressão, ou outros transtornos de afetividade (CUNHA AF e SANTOS VKR, 2018). A prevalência de depressão e ansiedade são maiores entre os estudantes de medicina se comparados à população mundial e ainda maior quando comparada à população mundial de mesma idade. Além disso, os estudantes de medicina brasileiros, quando comparados com a mesma população em outros países, tem maior prevalência de sintomas depressivos do que a média global (MAYER FB, et al., 2016). Mayer FB, et al. (2016), apontaram o reduzido acesso a acompanhamento psicológico e suporte de saúde mental como outro fator negativo vivenciado pelos acadêmicos, que seriam determinantes para o enfrentamento dos novos desafios exigidos na graduação.

Ademais, os estudantes de medicina também têm maior propensão de desenvolvimento do que Goldberg e Huxley denominaram de transtorno mental comum, que consiste em sintomas como alterações do sono, cansaço, irritabilidade, problemas de memória, dificuldade de concentração, queixas de ansiedade e depressão, além de queixas somáticas. Apesar de não constituírem um transtorno mental formal de acordo com os sistemas classificatórios e diagnósticos vigentes, proporcionam prejuízo a saúde mental e a qualidade de vida (SOUZA LPS, et al., 2017; CUNHA AF e SANTOS VKR, 2018). 
$\mathrm{Na}$ Psicopatologia atual, a afetividade conceitua-se como sendo um termo genérico, compreendendo vários elementos da vivência afetiva, sendo eles, o humor ou estado de ânimo, as emoções, os sentimentos, os afetos e as paixões (DALGALARRONDO P, 2008). O Afeto, em acepção mais ampla, representa qualquer estado de humor, sentimento ou emoção, podendo designar genericamente os elementos da afetividade, de modo que, alterações afetivas são empregadas muitas vezes em sentido amplo como sinônimo de alterações do humor. O humor, em sentido amplo, por sua vez, é em um elemento determinante da afetividade, que consiste no estado emocional basal, difuso, uma disposição afetiva de fundo que perpassa a experiência psíquica em sua totalidade, presente em um dado momento (BALDAÇARA L, et al., 2007). O humor se refere às emoções predominantes do indivíduo e suas alterações são substrato essencial no diagnóstico das alterações de afetividade, sendo exemplo delas, o humor deprimido, humor ansioso, humor irritado e humor hipertímico (BALDAÇARA L, et al., 2007; DALGALARRONDO P, 2008).

As alterações patológicas da afetividade, de diferente modo, designam um grupo de distúrbios mentais relacionados a vivência afetiva como um todo e são normalmente utilizados para se referir a patologias em seu conjunto de sintomas. Eles são separados em alterações de Humor, sendo, neste caso, o termo humor utilizado em seu sentido estrito de entidade nosológica, alterações da emoção e sentimento, e ansiedade. São exemplos de alterações da afetividade os transtornos de depressão, ansiedade, transtorno bipolar, disforia, irritabilidade patológica, fobia, terror (BALDAÇARA L, et al., 2007). Para auxiliar na difícil tarefa de tornar conceitos tão subjetivos quanto os descritas pelos psicopatologistas e para ajudar na interpretação dos sintomas na finalidade de facilitar o diagnóstico das alterações mentais, tais como as de afetividade, foram criados instrumentos que estabelecem critérios objetivos, sendo o mais utilizados deles o Manual Diagnóstico e Estatístico de Transtornos Mentais 5. a edição (DSM-V) (APA, 2014). Não obstante, os critérios de alteração do humor são tão fundamentais para o DSM-V, que são classificados como critérios obrigatórias para o diagnóstico dos transtornos de depressão, ansiedade, bipolar e disruptivo (APA, 2014).

O uso abusivo de medicamentos psicotrópicos, como os antidepressivos, ansiolíticos, antimaníacos, antipsicóticos e psicoestimulantes está em ascensão entre os universitários e também passou a ser objeto de interesse científico por sua grande disseminação no meio acadêmico, de modo que, tem se percebido um aumento do consumo dessas substâncias associada ao aumento da carga de cobrança e estresse a que são submetidos esses acadêmicos (MAJORI S, et al., 2017).

Nawaz H, et al. (2017), em análise do consumo de psicofármacos entre estudantes de medicina, constataram que as principais motivações para o uso dessas substâncias foram o estresse psicológico e a reduzida autoestima relacionada às suas competências acadêmicas. Trabalhos recentes apontam que 0 consumo dessas medicações psicotrópicas em boa parte das vezes tem sido realizada sem prescrição, em regime de automedicação, podendo ser por reutilização de prescrições médicas antigas, aquisição de medicamentos sem receita, compartilhamento dos medicamentos com pessoas próximas e reutilização tratamentos anteriores (NAWAZ H, et al., 2017; TERRA JUNIOR AT, 2018).

Entre os psicotrópicos, a classe mais utilizada para a diminuição da ansiedade e da tensão são os hipnóticos e sedativos, em especial os benzodiazepínicos (VARMA S, 2016). Divac N, et al. (2010), após estudo do uso de benzodiazepínicos em estudantes de medicina na Sérvia, constataram que os dois principais motivos para o seu consumo foram insônia e ansiedade, tendo relatado que ambos tinham impacto negativo na vida acadêmica e social. Um fármaco sedativo tem como principais ações a diminuição da atividade, a moderação da excitação e o relaxamento psicomotor do indivíduo. Já os hipnóticos agem sobre o sono produzindo sonolência e facilitando sua manutenção.

Diversas são as drogas hipnótico-sedativas, sendo os principais os benzodiazepínicos, os "compostos Z", os congêneres da melatonina e os barbitúricos, de modo que, todos esses fármacos possuem propriedades hipnóticos e sedativas em graus variados. Entretanto, foi apenas com o advento dos fármacos agonistas do receptor de benzodiazepínicos, conhecidos como benzodiazepínicos, e os novos agonistas, os "compostos Z", que forneciam ação no SNC de forma mais segura, sem o risco de provocar depressão nervosa generalizada, que essas classes de fármacos tiveram seu consumo popularizados com o objetivo de modulação do sono e de redução da ansiedade. 
A maioria dos fármacos hipnóticos-sedativos utilizados na atualidade atuam aumentando a afinidade do receptor GABA, facilitando a entrada de cloro nos neurônios e potencializando as vias de ação do sistema de transmissão gabaérgico, produzindo por sua vez a redução do estado de alerta, induzindo o sono, diminuindo a ansiedade e promovendo relaxamento muscular (BRUNTON LL, et al., 2018).

Por outro lado, apesar de a utilização de fármacos hipnóticos-sedativos ser bastante difundida, podem trazer efeitos adversos indesejáveis, como dependência química, alterações do sono, alterações cognitivas agudas e à longo prazo, distúrbios motores (VARMA S, 2016; NADER D e GOWING L, 2020). Além desses efeitos colaterais já conhecidos, a utilização dessas substâncias pode não só provocar alterações do humor como também precipitar transtornos como depressão e ansiedade, ou mesmo, agravá-los (ZULLIG KJ e DIVIN AL, 2012). Apesar de tais evidências, existem poucos estudos hoje que relacionam os efeitos adversos do uso de hipnóticos-sedativos em estudantes de medicina e ainda menos estudos que relacionam o uso desses medicamentos entre estudantes de medicina e os seus efeitos associados às alterações afetivas.

Desse modo, o presente estudo objetiva avaliar a relação entre o uso de hipnóticos-sedativos e o surgimento de queixas associadas a alterações do humor entre os alunos do primeiro ao sexto ano do curso de medicina de uma universidade particular de Sergipe, além de identificar o perfil de consumo desses medicamentos, compreender seus motivos precipitantes e avaliar o histórico de transtornos de afetividade entre os estudantes.

\section{MÉTODOS}

Trata-se de um recorte de um estudo clínico não experimental, exploratório, transversal, descritivo e de abordagem analítica qualiquantitativa. $O$ universo amostral foi constituído por todos os acadêmicos do curso de medicina de uma universidade particular localizada em um município do estado de Sergipe.

A amostra almejada foi de 254 alunos e teve seu cálculo baseado na fórmula de Gil, para populações finitas, que analisa o tamanho necessário da mesma (GIL AC, 2008). Essa amostra foi baseada em uma população finita de 812 alunos no total de graduandos em medicina das doze etapas, totalizando os 6 anos do curso, na prevalência extrapolada de $40 \%$ do consumo de drogas lícitas. Foi analisada a resposta de 281 alunos que efetivamente responderam ao questionário aplicado. O erro amostral foi de $5 \%$ com nível de confiança de $95 \%$.

Os critérios de inclusão foram estudantes de medicina de ambos os sexos, acima de 18 anos, devidamente matriculados em uma determinada universidade particular localizada no estado de Sergipe, em sua devida etapa cursada e que assinaram o termo de consentimento livre e esclarecido (TCLE), de acordo com as normas do Conselho Nacional de Saúde do Ministério da Saúde explicitadas na resolução 466/12. O trabalho foi iniciado somente após aprovação do Comitê de Ética em Pesquisa da Universidade Tiradentes (CEP/UNIT), com o parecer CAAE № 12088919.4.0000.5371.

A coleta de dados foi realizada no campus da referida universidade particular do estado de Sergipe. Após a assinatura do TCLE, foi aplicado um questionário específico que continha informações como idade, sexo, período que estavam cursando, tipo e a frequência de substâncias lícitas consumidas pelos estudantes, a relação do consumo com o desenvolvimento de queixas associadas a alterações do humor e possíveis transtornos psiquiátricos prévios diagnosticados (ansiedade, depressão, transtorno bipolar e transtorno obsessivo compulsivo). Os dados obtidos foram sigilosos e caso houvesse desejo do respondente e necessidade, eles poderiam ser orientados e encaminhados para atendimentos especializados.

Os dados coletados foram tabulados no Programa Microsoft Excel e analisados pela estatística descritiva. As variáveis categóricas foram descritas por meio de frequência absoluta e relativa percentual. As variáveis contínuas foram descritas por meio de média e desvio padrão. A aderência das variáveis contínuas a distribuição normal foi testada por meio do teste de Shapiro-Wilk. As diferenças nas medidas de tendência central foram testadas por meio do teste $\mathrm{T}$ para amostras independentes (normal). O nível e significância adotado foi de $5 \%$ e software utilizado foi o R Core Team 2020. 


\section{RESULTADOS}

Nesse estudo foram avaliados 281 estudantes do curso de medicina de uma universidade particular do estado de Sergipe, sem exclusões. A média de idade obtida foi de 22,8 anos com maioria do sexo feminino ( $n=189 ; 67,3 \%)$. Do total de estudantes que responderam ao questionário, $107(38,1 \%)$ relataram já ter consumido hipnóticos-sedativos. A maioria dos graduandos que relataram utilizar esses fármacos psicotrópicos eram do sexo feminino, o que representa um uso 2,5 vezes maior entre as estudantes de medicina em comparação com os do sexo masculino. Por sua vez, a média de idade encontrada entre os estudantes que relataram já ter feito uso dessas drogas foi semelhante à dos estudantes que participaram da pesquisa de modo geral (Tabela 1).

Tabela 1 - Perfil epidemiológico do uso de hipnóticos-sedativos em estudantes de medicina de uma universidade particular de Sergipe.

\begin{tabular}{lll}
\hline Variável & Média (anos) & \\
\hline Idade & 22,4 & \\
\hline & $\mathbf{N}$ & Porcentagem \\
\hline Sexo & & \\
\hline Masculino & 30 & $28 \%$ \\
Feminino & 77 & $72 \%$ \\
\hline Uso de Hipnóticos-Sedativos nos últimos 3 meses & & \\
\hline Nenhuma & 47 & $43,9 \%$ \\
1 ou 2 vezes & 31 & $29 \%$ \\
Mensalmente & 11 & $10,3 \%$ \\
Semanalmente & 10 & $9,3 \%$ \\
Diariamente & 8 & $7,5 \%$ \\
\hline Total & $\mathbf{1 0 7}$ & $\mathbf{1 0 0} \%$ \\
\hline
\end{tabular}

Fonte: Fraga RRA, et al., 2020. Legenda: N: número de casos.

Quanto aos aspectos motivacionais para o uso dos hipnóticos-sedativos, o principal fator que motivou o consumo foi a redução do estresse, representando 47,7\% ( $p$-valor: $<0,001$ ). 2,8\% dos estudantes relataram utilizar esses psicotrópicos para fins de diversão e porcentagens similares relataram utilizar para fins de socialização e necessidade de melhora do desempenho acadêmico, não tendo, esses, significância estatística para o presente estudo. Além disso, 13,1\% relataram fazer uso por queixas relacionadas a condições álgicas

A avaliação da história psiquiátrica se deu de forma direta, tendo sido os participantes questionados se já haveriam tido algum diagnóstico de transtorno de psiquiátrico, de modo que, entre os estudantes que relataram ter feito uso de hipnóticos-sedativos, o transtorno de ansiedade foi o que obteve maior prevalência, com 60 casos, seguido pela depressão, com 29 casos (Tabela 3).

Tabela 2 - Motivação para o consumo de hipnóticos-sedativos por estudantes de medicina de uma universidade particular de Sergipe.

\begin{tabular}{lcc}
\hline Motivação & $\mathbf{N}$ & p-valor \\
\hline Redução de estresse & 51 & $<0,001$ \\
Diversão & 3 & 0,308 \\
Socialização & 3 & 0,026 \\
Necessidade de melhora do desempenho acadêmico & 4 & 0,010 \\
Cefaleia ou outras condições dolorosas & 14 & 0,005 \\
Outras & 46 & $<0,001$ \\
\hline
\end{tabular}

Fonte: Fraga RRA, et al., 2020. Legenda: N: número de casos; p-valor: significância estatística.

Em relação à associação entre o uso de hipnótico-sedativos e o relato do desenvolvimento de queixas associadas a alterações do humor, especificamente ansioso e deprimido, o presente estudo se utilizou dos critérios obrigatórios que se referem a mudanças do humor dos principais transtornos de afetividade presentes no Manual Diagnóstico e Estatístico de Transtornos Mentais 5. a edição, para servirem como características 
sintomatológicas objetivas (APA, 2014). Especificamente para a queixa relativa à ansiedade, os participantes foram indagados sobre a aparição posterior ao uso de hipnótico/sedativo de relato de "medo, ansiedade e preocupação excessiva na maioria dos dias", enquanto que para queixa relativa à depressão, os participantes foram indagados se "deprimido ou desinteressado nas atividades na maior parte do dia" (Tabela 4).

Tabela 3 - Relação entre estudantes do curso de medicina de uma universidade particular de Sergipe que faziam uso de hipnóticos-sedativos e histórico de transtornos de afetividade diagnosticados.

\begin{tabular}{llll}
\hline Variável & \multicolumn{3}{c}{ Uso de hipnóticos-sedativos } \\
\hline Transtorno Diagnosticado & Sim (\%) & Não (\%) & p-valor \\
\hline Depressão & 27,1 & 8 & $<0,001$ \\
Ansiedade & 56,1 & 26,4 & $<0,001$ \\
Transtorno Disruptivo & 0 & 0 & \\
Transtorno Bipolar & 3,7 & 0 & 0,010 \\
\hline
\end{tabular}

Fonte: Fraga RRA, et al., 2020. Legenda: p-valor: significância estatística.

Tabela 4 - Relação entre estudantes do curso de medicina de uma universidade particular de Sergipe que faziam uso de hipnóticos-sedativos e queixas associadas à alteração do humor após o consumo.

\begin{tabular}{cccl}
\hline Variável & \multicolumn{3}{c}{ Uso de hipnóticos-sedativos } \\
\hline Queixas relatadas após o uso & Sim(\%) & Não(\%) & p-valor \\
\hline Humor persistentemente elevado, expansivo ou irritável & 35,5 & 17,8 & 0,001 \\
Irritado, zangado ou com explosões de raiva frequentes & 31,8 & 13,8 & $<0,001$ \\
Deprimido ou desinteressado nas atividades na maior parte do dia & 38,3 & 17,8 & $<0,001$ \\
Medo, ansiedade e preocupação excessivos & 50,5 & 16,1 & $<0,001$ \\
\hline
\end{tabular}

Fonte: Fraga RRA, et al., 2020. Legenda: p-valor: significância estatística.

\section{DISCUSSÃO}

A vida acadêmica dos estudantes universitários exige dedicação de muitas horas de estudo, especialmente no curso de medicina. Além da graduação, a rotina médica é considerada atividade de alta pressão e tensão psicológica, o que influencia no desempenho dos estudantes. Muitos desses estudantes, para suportar a alta carga a que são submetidos diariamente, o estresse e o cansaço, utilizam medicamentos hipnóticos-sedativos para atenuar a ansiedade e preocupação gerada (RONCERO C, et al., 2015; LUNA IS, et al., 2018). Moutinho ILD, et al. (2019), apresentaram que a prevalência do uso de sedativos ao longo de dois anos entre os estudantes de medicina em uma universidade no estado de São Paulo foi equivalente a $17,1 \%$.

Nawaz H, et al. (2017), constataram uma prevalência de uso de hipnóticos-sedativos ao longo da vida no mesmo grupo populacional em universidade do Paquistão de 32\%. Já Motyka A, et al. (2007), em seu estudo com acadêmicos de medicina na Polônia, apresentaram prevalência ainda maior de consumo, apresentando $38 \%$ de consumo entre os estudantes. No presente estudo, os dados de prevalência do consumo de hipnóticos-sedativos se aproximaram dos obtidos no estudo polonês, de modo que, dos 281 estudantes avaliados, $38,1 \%$ relataram já haver feito uso de hipnóticos-sedativos, sendo que, $21,3 \%$ relataram uso nos últimos 3 meses.

No tocante à relação de consumo entre homens e mulheres, assim como nessa pesquisa, vários são os estudos que demonstraram uma prevalência de consumo maior entre as mulheres, com relação homem:mulher de 1:2 (MANCEVSKA S, et al., 2008; RONCERO C, et al., 2015; LUNA IS, et al., 2018; MOUTINHO ILD, et al., 2019). Os motivos para tal, poderiam estar relacionados à tendência de as mulheres apresentarem maior ansiedade e sofrimento em situações estressantes, além de pior percepção de seu desempenho acadêmico, se comparada aos seus colegas de curso do outro gênero (PARO HBMS, et al., 2019). O presente estudo, por sua vez, chegou a igual constatação, de modo que, encontrou uma relação de uso de hipnóticos-sedativos entre homem:mulher de aproximadamente 1:2,5. 
Ansiedade, depressão, esgotamento, podem ser manifestações psicológicas da exposição crônica a fatores estressores, de modo que, muitos estudantes acabam recorrendo ao uso de ansiolíticos para combater o estresse e suas complicações (AL-SAYED AA, et al. 2014).

Estresse é apontado como um dos principais fatores motivacionais para o uso de psicofármacos em diversos estudos e demonstra o impacto da pressão acadêmica a que é submetido o estudante de medicina (STONE AM e MERLO LJ, 2010; NAWAZ H, et al., 2017). Nawaz H, et al. (2017), pontuaram que o estresse psicológico foi o principal motivo para o uso de psicofármacos, de modo que, $49,3 \%$ dos estudantes de medicina alegaram fazer uso desses fármacos por esse motivo.

Divac N, et al. (2010), analisando os motivos para uso de benzodiazepínicos, encontraram que 30\% dos estudantes de medicina que consumiram este tipo de hipnótico-sedativo, foram por queixas relacionadas ao estresse acadêmico e a ansiedade gerada. Quando comparado aos motivos do uso de psicofármacos de modo geral pelos estudantes de medicina, os dados do presente estudo mostram-se consistentes com a literatura, tendo encontrado, em $47,7 \%$ dos casos, o estresse como principal fator motivador para o uso de hipnótico-sedativos, entretanto, ao se comparar com o estudo sérvio de Divac N, et al. (2010), que analisa especificamente a classe dos benzodiazepínicos, este estudo demonstrou porcentagem maior de estudantes que fizeram uso dos hipnóticos-sedativos motivados por estresse acadêmico.

Quando avaliada a história pregressa relacionada à saúde mental dos estudantes que participaram deste estudo, a relação entre o uso de hipnóticos-sedativos e o diagnóstico prévio de alterações de afetividade trouxe estreita correlação, demonstrando que o diagnóstico de transtornos como ansiedade e depressão tiveram alta prevalência nesse grupo. A análise desse dado aponta para a influência dos transtornos de afetividade, em especial depressão e ansiedade, sobre o uso de psicofármacos, sendo fatores de risco para o abuso de hipnóticos-sedativos (BEAUFORT IN, et al., 2017; ZULLIG KJ e DIVIN AL, 2012).

O presente estudo demonstrou alta prevalência de diagnóstico de ansiedade e depressão entre os estudantes que relataram ter feito uso de hipnóticos-sedativos, com $56,1 \%$ diagnosticados com ansiedade e $27,1 \%$ com depressão. Se analisada a população que não fazia uso de tais substâncias, a porcentagem foi de $26,4 \%$ e $8 \%$ de estudantes que relataram histórico de transtornos de ansiedade e depressão, respectivamente. Zullig KJ e Divin AL (2012), relataram que estudantes de medicina que faziam uso de sedativos teriam 1,36 vezes mais chances de apresentar sintomas depressivos.

Zullig KJ e Divin AL (2012), salientam que apesar de os hipnóticos-sedativos serem largamente usados como tratamento de sintomas de ansiedade e depressão, eles podem além de não provocar o efeito desejado de redução dos sintomas, também precipitar, ou mesmo exacerbar, essas alterações afetivas, principalmente se usados por longos períodos ou em regime de automedicação. Quando analisada a relação entre uso de hipnóticos-sedativos e o desenvolvimento de alterações do humor, foram encontrados sintomas de ansiedade e depressão, tendo, o presente estudo, evidenciado significância estatística entre os dados obtidos (p-valor $<0,001)$. A porcentagem de estudantes de medicina que após o uso de tais substâncias desenvolveram sintomas de medo, ansiedade ou preocupação excessiva foi de $50,5 \%$, e os que se apresentaram deprimidos ou desinteressados pelas atividades diárias na maior parte do dia foi de $38,3 \%$.

Mancevska S, et al. (2008) e Reynolds A (2019), em seus respectivos estudos, compararam o uso de sedativos com a prevalência de sintomas de ansiedade e depressão em estudantes de medicina, entretanto, sem estabelecer associação significativa entre os dados. Por sua vez, Walters KS, et al. (2018), pontua associação significante entre o uso de hipnóticos-sedativos com a apresentação de sintomas depressivos ( $p$ valor $<0,001$ ), sem, no entanto, estabelecer significância estatística quando analisada a associação com sintomas de ansiedade. Apesar de o presente estudo não ter tido como objetivo o diagnóstico de transtornos de afetividade descritos pelos critérios do DSM-V, ele constata o desenvolvimento de alterações do humor após o uso de hipnóticos-sedativos, o que demonstra os efeitos negativos do uso dessas substâncias na vivência afetiva, não obstante, são necessários mais estudos para servirem de comparação afim de melhor compreender a relação entre o uso de hipnóticos-sedativos entre estudantes de medicina e o desenvolvimento de alterações do humor após sua utilização. 


\section{CONCLUSÃO}

$\mathrm{O}$ avanço das faculdades de medicina, e o aumento vertiginoso do conhecimento científico médico acumulado, exigem cada vez mais estudo e dedicação dos estudantes de medicina. Essa intensa carga estressora a que são submetidos é um importante fator de risco para o desenvolvimento de alterações de ordem psíquica e consumo de hipnóticos-sedativos. O presente estudo apresentou alta prevalência do consumo de hipnóticos-sedativos em acadêmicos de medicina, sendo o estresse o seu principal motivador, e evidenciou a existência de associação entre o consumo dessas drogas e o desenvolvimento de alterações do humor. Resultados do presente estudo indicam a necessidade das escolas médicas de instituírem programas de conscientização acerca dos riscos envolvidos no consumo de hipnóticos-sedativos.

\section{REFERÊNCIAS}

1. AL-SAYED AA, et al. Sedative Drug Use among King Saud University Medical Students: A Cross-Sectional Sampling Study. Depression research and treatment. Hindawi. 2014, 3: 378738.

2. AMERICAN PSYCHIATRIC ASSOCIATION. Manual Diagnóstico e Estatístico de Transtornos Mentais: DSM-5. 5 ed. Porto Alegre. Artmed. 2014, 948p.

3. BALDAÇARA L, et al. Humor e afeto. Como defini-los?. Arquivos Médicos dos Hospitais e da Faculdade de Ciências Médicas da Santa Casa de São Paulo. 2017, 52(3): 108-113.

4. BEAUFORT IN, et al. The Depression, Anxiety and Stress Scale (DASS-21) as a Screener for Depression in Substance Use Disorder Inpatients: A Pilot Study. European Addiction Research. December 2017, 23(5): $260-268$.

5. BRUNTON LL, et al. A. As Bases Farmacológicas da Terapêutica de Goodman \& Gilman. 13. ed. Rio de Janeiro. McGraw-Hill/Artmed. 2018, 1760p.

6. CANDIDO FJ, et al. The use of drugs and medical students: a literature review. Revista da Associação Médica Brasileira. Maio 2018, 64(5): 462-468.

7. CUNHA AF, SANTOS VKR. Avaliação de transtornos mentais comuns em estudantes de Medicina do Campus Lagarto: prevalência e fatores associados, 41f, 2018.Trabalho de Conclusão de curso - Graduação em Medicina. Universidade Federal de Sergipe, Lagarto, 2018.

8. DALGALARRONDO P. sicopatologia e semiologia dos transtornos mentais. 2. ed. Porto Alegre. Artmed. 2018, 438p.

9. DIVAC N, et al. P03-204 - A cross-sectional pilot study of benzodiazepines use among medical students in Serbia. European Psychiatry. December 2010, 25(1): 1270.

10. FALLAH G et al. Stimulant use in medical students and residents requires more careful attention. Caspian $\mathrm{J}$ Intern Med. 2018, 9(1): 87-91.

11. FERRAZ L, et al. Substâncias psicoativas: o consumo entre acadêmicos de uma universidade do sul do Brasil. Momento. Diálogos em Educação. 2018, 27(1): 371-386.

12. GIL, AC. Métodos e técnicas de pesquisa social. São Paulo. Atlas. 2008.

13. LUNA IS, et al. Consumo de psicofármacos entre alunos de medicina do primeiro e sexto ano de uma universidade do estado de São Paulo. Colloquium Vitae. 2018, 10(1): 22-28.

14. MAJORI S, et al. Brain doping: stimulants use and misuse among a sample of Italian college students. Journal of preventive medicine and hygiene. 2017, 58(2): 130.

15. MANCEVSKA S, et al. Depression, anxiety and substance use in medical students in the Republic of Macedonia. Bratisl Lek Listy. 2008, 109(12): 568-572.

16. MAYER FB, et al. Factors associated to depression and anxiety in medical students: a multicenter study. BMC Medical Education. 2016, 16(1): 282.

17. MORGAN HL, et al. Consumo de Estimulantes Cerebrais por Estudantes de Medicina de uma Universidade do Extremo Sul do Brasil: Prevalência, Motivação e Efeitos Percebidos. Revista Brasileira de Educação Médica. 2017, 41(1): 102-109.

18. MOTYKA A, et al. Consumption of psychoactive substances - awareness of health consequences among the students of university schools in Poznan. Przegl Lek. 2007, 64(10): 808-810.

19. MOUTINHO ILD, et al. Prevalence, Incidence, and Factors Associated with Substance Use Among Medical Students. Journal of Addiction Medicine. 2019, 13(4): 295-299.

20. NADER D, GOWING L. Is Long-Term Benzodiazepine Use a Risk Factor for Cognitive Decline? Results of a Systematic Review. Journal of addiction. Hindawi. 2020 Jan 24:1569456.

21. NAWAZ H, et al. Use of psychoactive drugs among medical undergraduates in Abbottabad. Journal of Ayub Medical College. Abbottabad. 2017, 29(4): 599-603.

22. PARO HBMS, et al. Qualidade de vida do estudante de medicina: o ambiente educacional importa?. Revista de Medicina da Universidade de São Paulo. São Paulo. 2019, 98(2):140-147.

23. PIRES MS, et al. O uso de substâncias psicoestimulantes sem prescrição médica por estudantes universitários. Revista Científica Fagoc - Saúde. 2018, 3(2): 22-29.

24. PUTHRAN R, et al. Prevalence of depression amongst medical students: a meta-analysis. Medical Education. $31 \mathrm{Mar}$ 2016, 50(4): 456-468. 
25. QUEK TT, et al. The Global Prevalence of Anxiety Among Medical Students: A Meta-Analysis. International Journal of Environmental Research and Public Health. 2019, 16, 15, 2735.

26. RAFIQUE N, et al., Comparing levels of psychological stress and its inducing factors among medical students. Journal of Taibah University Medical Sciences. December 2019, 14(6): 488-494.

27. REYNOLDS A. Is non-medical use of prescription sedatives and sleeping pills associated with symptoms of depression, anxiety, and stress in undergraduate university students. Master of Health Sciences in Community, Public and Population Health. Oshawa, Ontario, Canada; University of Ontario Institute of Technolog; 1-Jul-2019.

28. RONCERO C, et al. Substance use among medical students: A literature review 1988- 2013. Actas Espanolas de Psiquiatria. 2015, 43(3): 109-121.

29. SOUZA LPS, et al. Prevalência de transtornos mentais comuns em adultos no contexto da Atenção Primária à Saúde. Revista Portuguesa de Enfermagem de Saúde Mental. 2017 Dezembro, 18: 59-66.

30. STONE AM, MERLO LJ. Attitudes of college students toward mental illness stigma and the misuse of psychiatric medications. Journal of Clinical Psychiatry. 2011 Feb, 72(2): 134-139.

31. TERRA JÚNIOR AT. Perfil dos universitários da área da saúde quanto ao uso de substâncias psicoativas na cidade de Ariquemes - RO. Tese de Doutorado - Programa de Pós-graduação em Clínica Cirúrgica. Faculdade de Medicina de Ribeirão Preto - USP, Ribeirão Preto, 2018.

32. WALTERS KS, et al. Substance Use, Anxiety, and Depressive Symptoms Among College Students. Journal of Child \& Adolescent Substance Abuse. 2018, 27(2): 103-111.

33. VARMA S. Benzodiazepines and hypnotics. Medicine. 2016, 44(12): 764-767.

34. VOLPE U, et al. Mental health and wellbeing among Italian medical students: a descriptive study. International Review of Psychiatry. 2019, 31(7): 569-573.

35. ZULLIG KJ, DIVIN AL. The association between non-medical prescription drug use, depressive symptoms, and suicidality among college students. Addictive Behaviors. 2012, 37(8): 890-899. 